\title{
Anatomical Nature of Radicular Leg Pain Analyzed by Clinical Findings
}

\author{
Yuzuru Takahashi, Jiro Hirayama, Seiji Ohtori, \\ Toshiyuki Tauchi and Kazuhisa Takahashi \\ Department of Orthopaedic Surgery, School of Medicine, \\ Chiba University, Chiba, Japan
}

( Received 11 January 2000, Accepted 6 June 2000 )

\begin{abstract}
Physical and neurological data of 127 patients with radicular leg pain due to lumbar disc herniation were retrospectively examined to elucidate the anatomical nature of radicular leg pain. The spatial coincidence and qualitative relationship of spontaneous pain to mechanical pain sensation of the skin and muscles were analyzed. The rate of spatial coincidence was calculated between spontaneous pain and cutaneous and muscular pain sensation. The qualitative relationship was investigated by relating the intensity of spontaneous pain to the grade of cutaneous and muscular mechanical pain sensation. The spatial coincidences of spontaneous pain to abnormal cutaneous pain sensation was significantly lower than that to muscle tenderness. The grade of muscle tenderness was significantly increased with the intensity of spontaneous pain, whereas cutaneous mechanical pain sensation had no qualitative relationship with spontaneous pain. Radicular leg pain in lumbar disc herniation was closely associated to muscles, suggesting that it is perceived in muscles.
\end{abstract}

Key words : radicular leg pain, lumbar disc herniation, pain, hyperalgesia, cutaneous pain sensation, muscle tenderness

\section{INTRODUCTION}

Patients with lumbar intervertebral disc disorders often report pain in the lower extremity. Several hypotheses have been addressed regarding the mechanism of pain associated with lumbar disc disorders ${ }^{33)}$. Leg pain associated with lumbar disc disorder may arise from referred pain mechanisms of degenerated discs ${ }^{30)}$, or may be produced by reflex spasm of leg muscles ${ }^{27,30)}$ and by reflex activation of sympathetic efferent functions ${ }^{16)}$. However, neuro- pathic pain mechanisms generated by mechanical and chemical irritation of spinal nerve roots and dorsal root ganglia by the herniated disc ${ }^{12,28)}$ is currently a predominant notion endorsed by many surgical evidence.

Pain due to lumbar spinal-nerve-root involvement (radicular pain) would be perceived in the structures innervated by injured sensory fibers contained in the involved nerve root, and generically termed radicular 'leg' pain. However, little attention has been paid to the anatomical components of radicular leg pain. Which structures of the leg are really painful? 
Table 1 Clinical data of the 127 patients

\begin{tabular}{|c|c|c|c|c|}
\hline Total number & & 127 & & \\
\hline Gender & male 75 ; female 52 & & & \\
\hline Age & $12 \sim 72(33.4+/-14.2 ;$ & mean & + & $(-$ S.D. $)$ \\
\hline \multirow[t]{3}{*}{ Level of disc herniation (MRI) } & L4/5 & 61 & ( & $48 \%)$ \\
\hline & $\mathrm{L} 5 / \mathrm{S}$ & 44 & ( & $35 \%)$ \\
\hline & $\mathrm{L} 4 / 5$ and $\mathrm{L} 5 / \mathrm{S}$ & 22 & ( & $17 \%)$ \\
\hline \multirow[t]{6}{*}{ Symptoms and signs } & spontanous pain & 127 & ( & $100 \%)$ \\
\hline & positive SLR & 99 & ( & $78 \%)$ \\
\hline & sensory change & 93 & ( & $73 \%)$ \\
\hline & muscle tenderness & 57 & ( & $45 \%)$ \\
\hline & motor weakness & 45 & ( & $35 \%)$ \\
\hline & reduced ATR & 39 & ( & $31 \%)$ \\
\hline
\end{tabular}

MRI: magnetic resonance image, SLR: straight leg raising test, ATR:

Achilles tendon reflex. Sensory change: cutaneous hyper- and hypo-

algesia. Moter weakness: reduced muscle power in the tibialis

anterior, extensor hallucis longus, triceps surae, or flexor hallucis

longus. Percentages in parentheses represent the incidence in the

127 patients.

Is it a specific tissue such as the skin, muscle, or bone? Or are all of these structures are equally painful?

We have routinely examined muscle tenderness in the back and lower extremities as a prevalent sign of lumbar radiculopathy. We reported that $55 \%$ of patients with lumbar disc herniation showed tenderness points in leg muscles, while the incidence of motor weakness and cutaneous sensory deficit in the lower extremity were $38 \%$ and $67 \%$, respectively ${ }^{35)}$. In contrast, cutaneous hyperalgesia seemed to be conspicuously rare in lumbar disc herniation.

A tissue with ongoing pain often presents local hyperalgesia regardless of the pathogenesis of pain. It is reported that experimentally induced pain is associated with mechanical hyperalgesia, by periph- eral and central nervous mechanisms ${ }^{8,36)}$. Therefore, the anatomical nature of radicular leg pain could be clarified by examining the pain sensation of associated tissues in painful areas.

To analyze the anatomical components of radicular leg pain, we investigated the spatial coincidence and qualitative relationship between spontaneous pain and mechanical pain sensation in the skin and muscles from clinical data of patients with unilateral leg pain due to lumbar disc herniation.

\section{CLINICAL MATERIALS AND METHODS}

\section{Patients}

The physical and neurological records at the first examination of 127 patients with unilateral leg pain due to lumbar disc herniation were retrospec- 
tively investigated. Radicular leg pain was diagnosed both radiologically and neurologically; the patients were demonstrated to have herniated $\operatorname{disc}(\mathrm{s})$ at either L4/5 or L5/S1 levels on magnetic resonance images and showed at least one of the following symptoms and signs indicating the impairment of L5 or S1 spinal nerve roots : weakness of muscles relevant to the ankle and big toe motions, cutaneous hypoalgesia in L5 or S1 dermatomes, reduced Achilles tendon reflex, or positive response in straight leg raising test. Thus, cases of referred leg pain were not included. Clinical data of the patients are presented in the Table 1. Seventy-five were men and 52 were women. Ages ranged from 12 to 72 years (mean 33.4 years).

\section{Examinations}

Physical and neurological examinations were performed by a single examiner (Y.T.) for all the patients. The location of spontaneous pain at rest or during motion was drawn on the body chart by the physician based on vocal information and indication given by the patient. Symptoms expressed only as 'itai' (painful) in Japanese were included as spontaneous pain ; abnormal sensations which were expressed in words other than itai, and may correspond to paresthesia, dysesthesia and numbness in English were excluded. The intensity of spontaneous pain was graded as none (denoted as 0 ), weak or occasional $(+1)$, strong or continuous $(+2)$.

Cutaneous mechanical pain sensation was examined by the standard pin prick test and compared to the response in the corresponding area of the contralateral, unaffected limb. Cutaneous pain sensation was graded as hyperalgesia (+1), normal (0), weak hypo-algesia $(-1)$, and strong hypo-algesia $(-2)$; grades $+1,-1$, and -2 were considered to be abnormal.

Muscle tenderness was examined manually via the thumb of the examiner at so called "motor point" of each muscle, because manual pressure evoked most intense pain at these points within a muscle as Gunn et al. described ${ }^{11)}$. Muscle tenderness was examined initially in the unaffected limb and the minimum force which induce painful sensation was determined. Then the same pressure force was
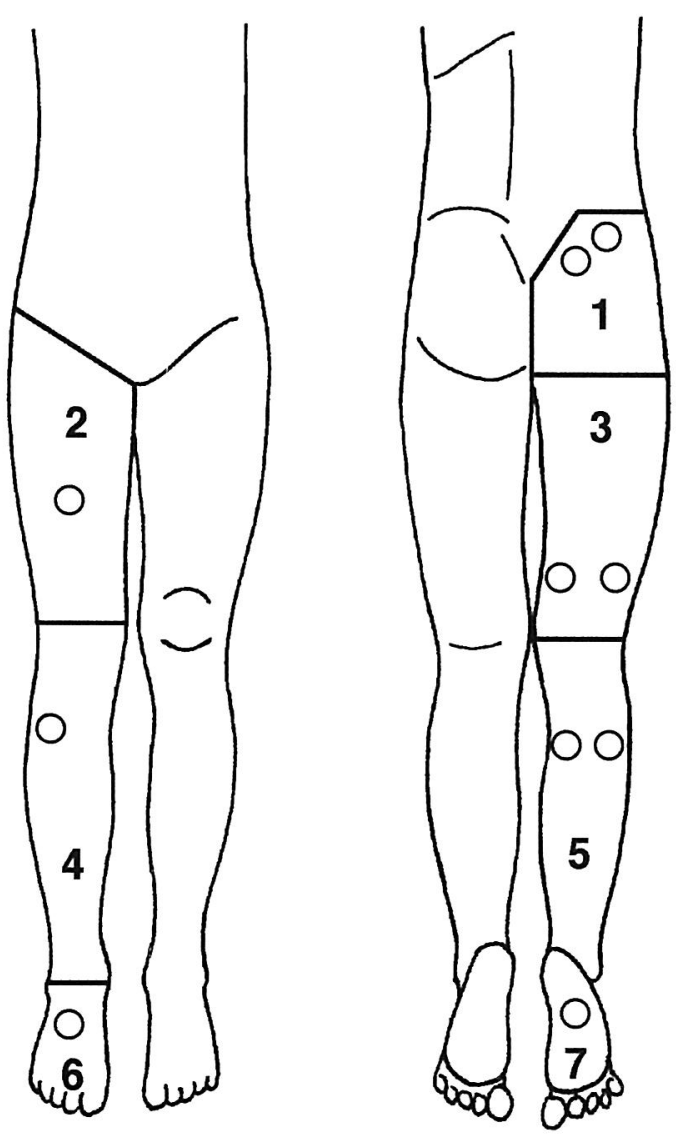

Fig. 1 Illustration showing the seven regions and the location of their motor points (open circles) where muscle tenderness was examined. 1 : the buttock, 2 : anterior thigh, $3:$ posterior thigh, $4:$ anterior leg, $5:$ posterior leg, $6:$ dorsum of the foot, and $7:$ sole.

applied to the corresponding point in the affected limb. In case that the intensity of evoked pain in the affect limb was identical in the unaffected limb, muscle tenderness of the affected limb was graded as normal (0). If evoked pain was severer and markedly severe occasionally with jump sign, it was graded as $(+1)$ and $(+2)$, respectively.

\section{Regions in the lower extremity}

To facilitate the analysis of the spatial coincidence and qualitative relationship between spontaneous pain and cutaneous and muscular mechanical pain sensation, the lower extremity was divided into the following seven regions : the buttock, anterior 
Table 2 Incidence of spontaneous pain, cutaneous abnormal pain sensation, muscle tenderness in the 127 cases

\begin{tabular}{lccc}
\hline Region & Spontaneous pain & Abnormal pain sensation \\
\cline { 3 - 4 } & & Skin & Muscle \\
\hline Buttock & $66 \%$ & $6 \%$ & $32 \%$ \\
Anterior thigh & $9 \%$ & $8 \%$ & $3 \%$ \\
Posterior thigh & $45 \%$ & $14 \%$ & $23 \%$ \\
Anterior leg & $11 \%$ & $30 \%$ & $6 \%$ \\
Posterior leg & $51 \%$ & $34 \%$ & $25 \%$ \\
Dorsal foot & $6 \%$ & $49 \%$ & $0 \%$ \\
Sole & $27 \%$ & $20 \%$ & $13 \%$ \\
\hline
\end{tabular}

thigh, posterior thigh, anterior leg, posterior leg, dorsum of the foot, and sole (Fig. 1).

Spontaneous pain and abnormal cutaneous pain sensation in a given region was determined to be positive when the symptom and sign were located within the region regardless of its size.

Muscle tenderness was examined on the motor points of the gluteus medius and the gluteus maximus muscles on the iliac alar in the buttock. The highest tenderness score represented the grade of muscle tenderness of the region. Likewise, muscle tenderness was examined in the quadriceps femoris in the anterior thigh, the semimembranosus, semitendinosus, and biceps femoris in the posterior thigh, the tibialis anterior in the anterior leg, the medial and lateral head of triceps surae in the posterior leg, the extensor tendons in the dorsal foot, and the flexor tendons and the flexor digitorum brevis muscle in the sole (Fig. 1).

\section{Analysis of data}

The spatial coincidence and qualitative relationship were calculated in a total of 889 (127 multiplied by 7$)$ regions. The spatial coincidence was expressed as the percentage of the sum of the number of regions with spontaneous pain and abnormal mechanical pain sensation (of the skin or muscles) and the number of regions without spontaneous pain and with normal mechanical pain sensation (of the skin or muscles) in the total 889 regions. The percentages of the grades of abnormal pain sensation in each grade of spontaneous pain were calculated to exhibit the qualitative relationship between spontaneous pain and abnormal pain sensation.

\section{Statistics}

Values related to the spatial correlation were statistically analyzed using the chi-square test. The qualitative correlation was analyzed non-parametrically by Kruskal-Wallis test. P values of less than 0.01 were considered to be significant.

\section{RESULTS}

\section{Patients}

The most frequent sign suggestive of radicular involvement was positive straight leg raising test (78\%). The incidence of abnormal cutaneous pain sensation and muscle tenderness was $93(73 \%)$, and 57 (45\%), respectively (Table 1).

\section{Spatial relationship}

Table 2 shows the incidence of spontaneous pain, abnormal cutaneous pain sensation, and muscle tenderness in each region. Spontaneous pain and muscle tenderness were frequent in the buttock, posterior thigh, and posterior leg, whereas the incidence of abnormal cutaneous pain sensation was high 
Table 3 Spatial relationship between spontaneous pain and cutaneous abnormal pain sensation

\begin{tabular}{lccc}
\hline Spontaneous pain & Cutaneous pain sensation \\
& & 0 & $-2,-1,+1$ \\
\hline $1,+2$ & $n=647$ & $\underline{499}$ & 148 \\
total & $n=889$ & 187 & $\underline{55}$ \\
\hline
\end{tabular}

Values represent the number of regions.

Table 4 Spatial relationship between spontaneous pain and muscle tenderness

\begin{tabular}{llcc}
\hline Spontaneous pain & Muscle tenderness \\
& & 0 & $+1,+2$ \\
\hline 0 & $n=647$ & $\underline{617}$ & 30 \\
$+1,+2$ & $n=242$ & 158 & $\underline{84}$ \\
\hline total & $n=889$ & 775 & 114 \\
\hline
\end{tabular}

Values represent the number of regions.

Table 5 Spatial coincidence between spontaneous pain and abnormal cutaneous pain sensation and muscle tenderness

\begin{tabular}{lcc}
\hline Relation to & $\begin{array}{r}\text { Cutaneous hyper or } \\
\text { Hypo-algeisa }\end{array}$ & $\begin{array}{c}\text { Muscle } \\
\text { tenderness }\end{array}$ \\
\hline coincidence & $554(62 \%)$ & $701(79 \%)$ \\
inconsistent & $335(38 \%)$ & $188(21 \%)$ \\
\hline total & 889 & 889 \\
\hline
\end{tabular}

Values represent the number of regions.

in distal regions.

No significant spatial correlation was found between spontaneous pain and abnormal cutaneous sensation ( $p=0.962$, Table 3). The overall spatial coincidence between the two factors was 554 $(=499+55) / 889=62 \%$. Significant spatial correlation was found between spontaneous pain to muscle tenderness $(p<0.0001$, Table 4$)$. The overall spatial coincidence between the two factors was 701 $(=617+84) / 889=79 \%$.

The value of coincidence was significantly higher in muscle tenderness (79\%) than in abnormal cutaneous pain sensation $(62 \%)(p<0.0001$, Table 5). Qualitative relationship

Cutaneous mechanical pain sensation had no qualitative relationship with spontaneous pain 


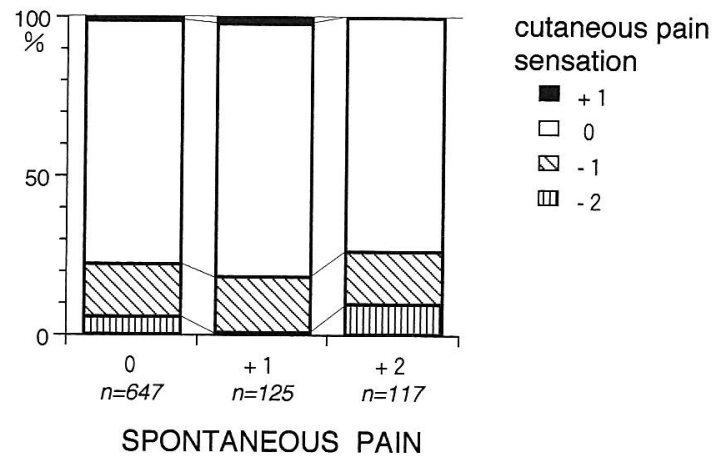

Fig. 2 Qualitative relationship between spontaneous pain and pain sensation of the skin.
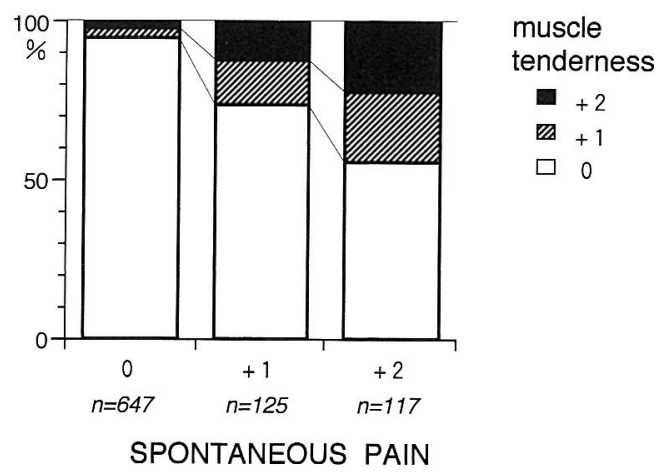

Fig. 3 Qualitative relationship between spontaneous pain and muscle tenderness.

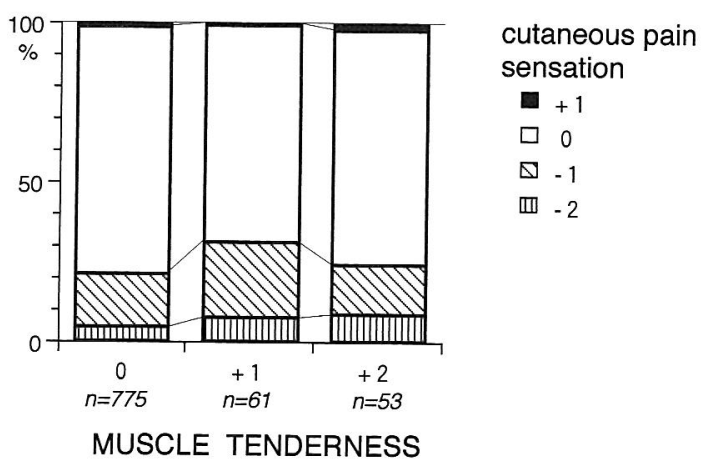

Fig. 4 Qualitative relationship between muscle tenderness and pain sensation of the skin.

( $p=0.4003$, Fig. 2 ). In contrast, muscle tenderness was significantly increased with the grade of spontaneous pain $(p<0.001$, Fig. 3). The muscle tenderness was unrelated to hyper and hypo-algesia of the overlying skin ( $p=0.4990$, Fig. 4).

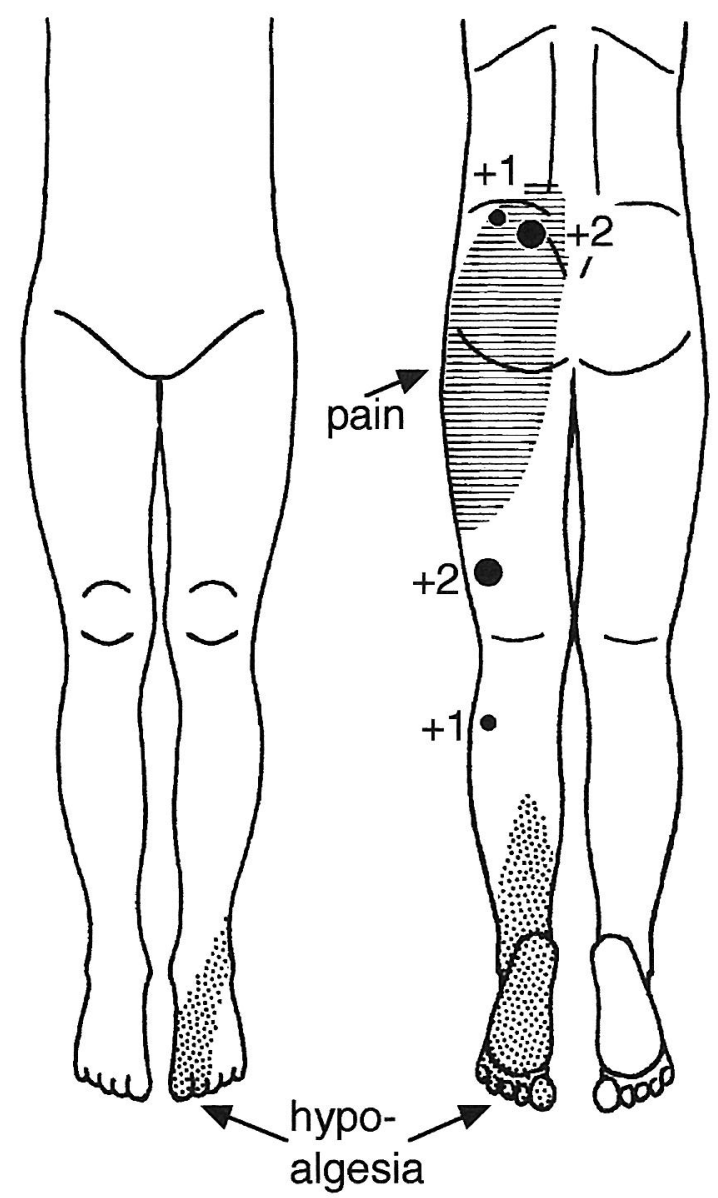

Fig. 5 A typical case of lumbar radiculopathy due to lumbar disc herniation at L5/S1. Hashed and dotted area show spontaneous pain and cutaneous hypo-algesia, respectively. Filled circles indicate the motor points with muscle tenderness. The size of the circle and the adjunct numeral represent the intensity of muscle tenderness.

\section{Case presentation (Fig. 5)}

Thirty years-old woman suffering from herniated intervertebral disc at L5/S1 level. Her chief complaint was pain in the left buttock and posterior thigh. The SLR test was positive with evoked thigh pain at 40 degrees. The tibialis anterior, extensor hallucis longus, and triceps surae muscle showed mild weakness (4/5 level with manual muscle test). Moderate hypo-algesia (7/10 level as compared in the contralateral side) was found in the L5 and S1 dermatomes within the foot and posterior leg regions. 
The gluteus medius and the lateral head of the triceps surae showed weak muscle tenderness and the gluteus maximus and the lateral hamstring muscles showed strong muscle tenderness with jump sign. This typical case clearly shows that spontaneous pain is more closely related to muscle tenderness than to cutaneous hypo-algesia.

\section{DISCUSSION}

Herniated intervertebral discs are not rare in asymptotic healthy subjects ${ }^{3,15}$. Even the SLR sign may be positive in the facet joint pain ${ }^{24)}$ and dorsal rami syndrome ${ }^{21)}$. Thus, there are no gold standards to diagnose lumbar radiculopathy based on clinical data. The current most strict criteria of lumbar radiculopathy are the reproduction of typical pain and subsequent reduction of the pain by spinal nerve infiltration, or visual verification of nerve root compression during surgery. However, it may be accepted by physician that combined with radiological and neurological evidence, lumbar radiculopathy can be diagnosed.

The present study elucidated that in lumbar radiculopathy, the incidence of muscle tenderness and spontaneous pain were high in the proximal and posterior areas of the lower extremities, whereas the incidence of abnormal cutaneous pain sensation was high in the distal areas. Subsequently, spontaneous pain was more spatially related to muscular pain sensation than to cutaneous pain sensation.

Disc herniations at L4/5 and L5/S levels potentially damage the L5 and S1 and occasionally L4 spinal nerve roots. Recently, we performed basic studies with respect to the arrangement of dermatomes and found that the shape of the dermatomes in the limbs tapers proximally ${ }^{32)}$. In humans, L4, L5, and S1 dermatomes are distributed mainly in the leg and foot, but sparsely in the buttock, due to the absence of dorsal cutaneous nerves in these segments ${ }^{4,10,34)}$. Contrary to dermatomes, the gluteus muscles which occupy most of the buttock area are innervated by the superior gluteal nerve consisting of L4-S1 spinal nerves ${ }^{20)}$. These neuroanatomical facts also suggest that the pain which appears in the buttock and the posterior side of the

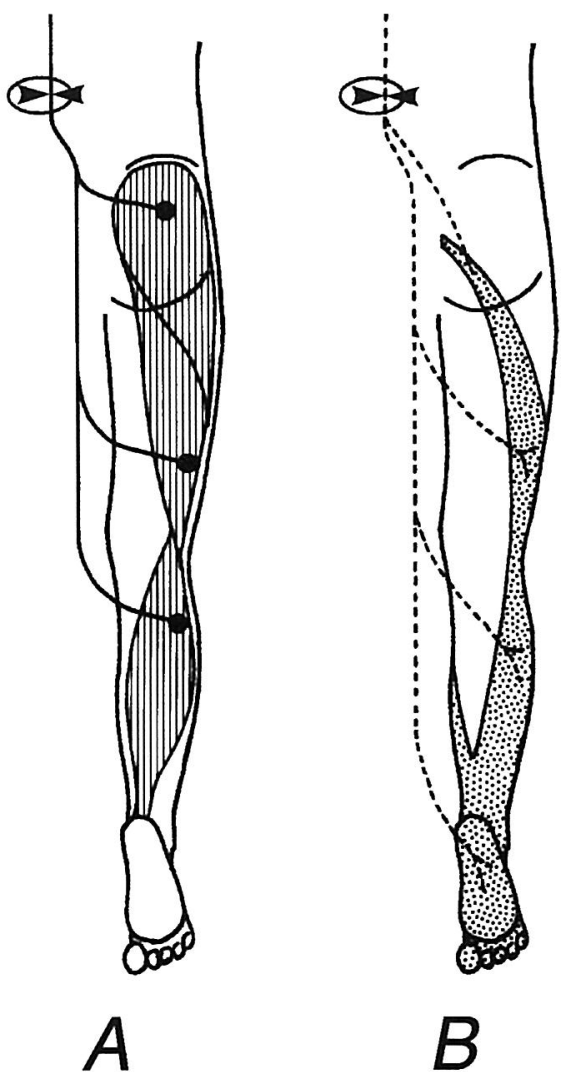

Fig. 6 Schematic drawing representing the concept of the pathogenesis of spontaneous pain and abnormal cutaneous pain sensation in spinal nerve root impairment at L5 and S1 segments. Arrowheads indicate the site of nerve root impairment due to disc herniation. A : Spontaneous pain is sensed in the muscles (hashed areas) innervated by the impaired nerve fibers (solid lines), and tenderness occurs at the motor point (filled circle) of the muscles. B : Abnormal cutaneous pain sensations are examined in the dermatome (dotted area) of the impaired nerve fibers to the skin (broken lines).

lower extremity follows the myotomal pattern (Fig. 6).

It is frequently observed that injuries and some inflammatory diseases of the skin and connective tissues result in local cutaneous hyperalgesia, and those of the joint develop immobility because of pain elicited within the normal range of motion. Experimentally, stimulation of human cutaneous 
sensory C-fibers induces pain and concomitant mechanical cutaneous hyperalgesia ${ }^{8,31,36)}$. In experimental arthritis, nociceptors in the joint capsule are sensitized in inflammatory conditions ${ }^{7,13)}$. Thus, the tissue where spontaneous pain is sensed frequently show local mechanical hyperalgesia in injuries and local inflammation.

Peripheral neuropathy presents various conditions of pain sensation in a painful area. In nerve injuries, the innervation area of an impaired nerve usually presents normo- or hypo-algesia ${ }^{29)}$. It is reported that about one-half of the patients with carpal tunnel syndrome show cutaneous hypo-algesia in the fingers ${ }^{6)}$. In contrast, in a severe condition of neuropathic pain like causalgia, painful areas present cutaneous hyperalgesia and immobility of the joint suggestive of hyperalgesia of the joint tissues ${ }^{5}$. Taking these facts into consideration, in a neuropathic pain syndrome, when tissues with and without hyperalgesia can be discriminated within a painful area, it could be stated that tissues presenting hyperalgesia are more likely to be those where spontaneous pain is perceived. The present results demonstrated that radicular leg pain was related to hyperalgesia of the muscle, not to that of the skin. Hence, radicular leg pain is likely to be perceived in muscles.

Recently, several 'neuropathic pain models' using rats have been addressed for the research of neuropathic pain ${ }^{2,19)}$. In these models, hyperalgesia is measured as an alternative index of the severity of neuropathic pain that can not be assessed in rats. The hyperalgesia rat model is recently also applied to studies of lumbar radiculopathy ${ }^{17,18)}$. However, as revealed in the present study, mechanical hyperalgesia was hardly reported from patients with lumbar disc herniation, indicating that the hyperalgesia rat model is inappropriate for typical lumbar radiculopathy. There are case reports of patients with lumbar disc herniation who showed causalgia-like symptoms in the foot ${ }^{1,9,14)}$. The hyperalgesia rat model may only reproduce extraordinary severe, but rare, conditions of lumbar radiculopathy.

In studies on pain distribution of low back pain patients, the pain drawing is preferably performed by patients themselves $22,23,25,26)$. Such studies are aimed to reveal a psychological, non-organic features of patients' complaints based on a drawing pattern. In contrast, we draw pain chart by ourselves because we need to record the location of pain as exact as possible in order to investigate the diagnostic value of pain location.

Methodologically, manual pressing can not discriminate whether evoked pain comes from the skin, connective tissue, or bones. Only cutaneous hyperalgesia could be ruled out from the present result (Fig. 4). The 'muscle tenderness' may be more accurately termed as 'mechanical hyperalgesia of deep somatic structures'.

Finally, the present results indicate that physical therapies essentially affect the skin, such as poultices and acupuncture, may be less effective to radicular leg pain. Therapies directly affect muscles would have more therapeutic effects.

\section{REFERENCES}

1) Ballard, E. M., Ellenberg, M. and Chodoroff, G., Reflex sympathetic dystrophy syndrome secondary to L5 radiculopathy, Arch. Phys. Med. Rehabil., 72 (1991) 595-597.

2) Bennett, G. J. and Xie, Y. K., A peripheral mononeuropathy in rat that produces disorders of pain sensation like those seen in man, Pain, 33 (1988) 87 107.

3) Boden, S. D., Davis, D. O., Dina, T. S., Patronas, N. J. and Wiesel, S. W., Abnormal magnetic-resonance scans of the lumbar spine in asymptomatic subjects, J. Bone Joint Surg. [Am], 72-A (1990) 403-408.

4) Bonica, J. J., Applied anatomy relevant to pain. In : J. J. Bonica (Ed.), The Management of Pain, Lea \& Febiger, Philadelphia and London, Second edn., 1990, pp133-158.

5) Bonica, J. J., Causalgia and other reflex sympathetic dystrophies. In : J. J. Bonica (Ed.), The Management of Pain, Lea \& Febiger, Philadelphia, London, Second edn., 1990, pp220-230.

6) Borg, K. and Lindblom, U., Diagnostic value of quantitative sensory testing (QST) in carpal tunnel syndrome, Acta Neurol. Scand., 78 (1988) 537-541.

7) Coggeshall, R. E., Hong, K. A. P., Langford, L. A., Schaible, H. G. and Schmidt, R. F., Discharge characteristics of fine medial articular afferents at rest and during passive movements of inflammed knee joints, 
Brain Res., 272 (1983) 185-188.

8) Culp, W. J., Ochoa, J., Cline, M. and Dotson, R.. Heat and mechanical hyperalgesia induced by capsaicin. Cross modality threshold modulation in human C nociceptors, Brain, 112 (1989) 1317-1331.

9) Dressler, D., Thompson, P. D., Gledhill, R. F. and Marsden, C. D., The syndrome of painful legs and moving toes, Movement Disorders, 9 (1994) 13-21.

10) Foerster, O., The dermatomes in man, Brain, 56 (1933) 1-39.

11) Gunn, C. C., Chir, B. and Milbrandt, W. E., Tenderness at motor points. A diagnostic and prognostic aid for low-back injury, J. Bone Joint Surg. [Am], 58-A (1976) 815-825.

12) Hasue, M., Pain and the nerve root. An interdisciplinary approach, Spine, 18 (1993) 2053-2058.

13) Heppelmann, B. and Pawlak, M., Sensitisation of articular afferents in normal and inflammed knee joints by substance P in the rat, Neurosci. Lett., 223 (1997) 97-100.

14) Ishizuka, K., Yasutake, S., Yoshino, M., Ishii, M. and Takeuchi, T., Intra- and extra foraminal lumbar disc herniation with symptoms of sympathetic derangement in the foot, Seikeigeka, 46 (1995) 1528-1531.

15) Jensen, M. C., Brant-Zawadzki, M. N., Obuchowski, N., Modic, M. T., Malkasian, D. and Ross, J. S., Magnetic resonance imaging of the lumabar spine in people without back pain, New Engl. J. Med., 331 (1994) 69-73.

16) Jinkins, J. R., Wittemore, A. R. and Bradley, W. G., The anatomic basis of vertebrogenic pain and the autonomic syndrome associated with lumbar disc extrusion, Am. J. Radiol., 152 (1989) 1277-1289.

17) Kawakami, M., Tamaki, T., Weinstein, J. N., Hashizume, H., Nishi, H. and Meller, S. T., Pathomechanism of pain-related behavior produced by allografts of intervertebral disc in the rat, Spine, 21 (1996) 2101-2107.

18) Kawakami, M., Weinstein, J. N., Chatani, K., Spratt, K. F., Meller, S. T. and Gebhart, G. F., Experimental lumbar radiculopathy. Behavioral and histologic changes in a model of radicular pain after spinal nerve root irritation with chromic gut ligatures in the rat, Spine, 19 (1994) 1795-1802.

19) Kim, S. H. and Chung, J. M., An experimental model for peripheral neuropathy produced by segmental spinal nerve ligation in the rat, Pain, 50 (1992) 355 363.

20) Kimura, J., Electrodiagnosis in diseases of nerve and muscle : principles and practice, F.A. Davis, Philadelphia, 1983, pp 17-26.
21) King, J. S. and Lagger, R., Sciatica viewed as a referred pain syndrome, Surg. Neurol., 5 (1976) 4650.

22) Ljüngren, A. E., Jacobsen, T. and Osvik, A., Pain descriptions and surgical findings in patients with herniated lumbar intervertebral discs, Pain, 35 (1988) 39-46.

23) Mann, N. H., Brown, M. D., Hertz, D. B., Enger, I. and Tompkins, J., Initial-impression diagnosis using low-back pain patient pain drawings, Spine, 18 (1993) 41-53.

24) Moony, V. and Robertson, J., The facet syndrome, Clin. Orthop., 115 (1976) 149-156.

25) Ohnmeiss, D. D., Vanharanta, H. and Ekholm, J., Degree of disc disruption and lower extremity pain, Spine, 22 (1997) 1600-1605.

26) Ransford, A. O., Cairns, D. and Moony, V., The pain drawing as an aid to the psychologic evaluation of patients with low-back pain, Spine, 1 (1976) 127 134.

27) Rosomoff, H. L., Do herniated discs produce pain? In : H. L. Fields, R. Dubner and F. Cervo (Ed.), Advances in Pain Research and Therapy, Raven Press, New York, edn., 1985, pp457-461.

28) Rydevik, B. and Olmarker, K., Pathogenesis of nerve root damage. In : M. I. V. Jayson (Ed.), The Lumbar Spine and Back Pain, Churchill Livingstone, Edinburgh, London, Melbourne, New York, Tokyo, Fourth edn., 1992, pp89-100.

29) Seddon, H., Surgical Disorders of the Peripheral Nerves, Nankodo, Tokyo, 1978, 45-51pp.

30) Simmons, J. W., Ricketson, R. and McMillin, J. N., Painful lumbosacral sensory distribution patterns : Embryogenesis to adulthood, Orthop. Rev., 22 (1993) 1110-1118.

31) Simone, D. A., Baumann, T. K. and LaMotte, R. H., Dose-dependent pain and mechanical hyperalgesia in humans after intradermal injection of capsaicin, Pain, 38 (1989) 99-107.

32) Takahashi, Y. and Nakajima, Y., Dermatomes in the rat limbs as determined by antidromic stimulation of sensory C-fibers in spinal nerves, Pain, 67 (1996) 197-202.

33) Takahashi, Y., Nakamura, S., Suseki, K., Takahashi, K. and Yamagata, M., Pain distribution in lumbar disc lesions, Rinsho Seikeigeka, 32 (1997) 69-75.

34) Takahashi, Y., Takahashi, K. and Moriya, H., Mapping of dermatomes of the lower extremities based on an animal model, J. Neurosurg., 82 (1995) 1030-1034.

35) Takahashi, Y., Takahashi, K., Yamagata, M., Ohtake, 
Y., Toyone, T., Morinaga, T. and Moriya, H., Tenderness points in the lower extremity in patients with lumbar radiculopathy, Rinsho Seikeigeka, 29 (1994) 867-872.
36) Torebjörk, H. E., Lundberg, L. E. R. and LaMotte, R. $H$. , Central changes in processing of mechanoreceptive input in capsaicin-induced secondary hyperalgesia in humans, J. Physiol., 448 (1992) 765-780.

Address for correspondence : Yuzuru Takahashi, M.D., Ph.D.

Department of Orthopaedic Surgery, School of Medicine, Chiba University

1-8-1 Inohana, Chuo-ku, Chiba 260-8677, Japan

Tel : +81-43-226-2117 Fax : +81-43-226-2116

E-mail : BXM06740@nifty.ne.jp 